Hodkinson, P. (2015) 'Bedrooms and Beyond: Youth, Identity and Privacy on Social Network Sites', New Media and Society, online before print, DOI:

$10.1177 / 1461444815605454$

Note - this document is the pre-publication version accepted by the journal. The published version can be found at http://nms.sagepub.com/content/early/2015/09/20/1461444815605454.abstract

\title{
Bedrooms and Beyond: Youth, Identity and Privacy on Social Network Sites
}

\author{
Paul Hodkinson
}

\section{Abstract}

This article considers young people's identities and privacy on social network sites through reflection on the analogy of the teenage bedroom as a means to understand such spaces. The notion therein of intimate personal space may jar with the scope and complexity of social media and, particularly, with recent emphasis on the challenges to privacy posed by such environments. I suggest, however, that, through increased use of access controls and a range of informal strategies, young people's everyday digital communication may not be as out of control as is sometimes inferred. Recent adaptations of the bedroom analogy indicate that social network sites retain intimacy and that their individual-centred format continues to facilitate the exhibition and mapping of identities. Though an awkward fit, I suggest the bedroom may still help us think through how social network sites can function as vital personal home territories in the midst of multi-spatial patterns of sociability. 


\section{Keywords}

Social network site; youth; bedroom; privacy; identity

\section{Introduction}

Participation in online cultures of sharing and interaction via social media is becoming increasingly ubiquitous and, arguably, compulsory among groups of young people in late capitalist societies (Robards, 2014; Marwick and boyd, 2014). And, particularly as a result of the evolution and diffusion of mobile multimedia technologies, such participation takes on an increasingly 'always on' character, whereby users' connection with others via such platforms forms a constant feature of everyday lives that traverse a range of physical settings. Against this context, discussion of the opportunities and challenges social media environments present for young people continues to feature prominently in academic research and the broader public sphere. Amongst other things, questions about the establishment of identity through performance and interaction, and about constraints, risks and challenges with respect to young people's social privacy', have continued to dominate such discourse.

Some have explored the spatial analogy of the teenage bedroom as a means to conceptualise the personalised orientation of social media and their particular affordances with respect to identity work and performance. Others have given greater weight to 
apparently public features of such environments, devoting particular attention to the ways they may compromise, confuse and complicate young people's privacy and, as a consequence, render more difficult the establishment of identity in social worlds. Sometimes public spatial analogies, such as parks and shopping malls are referred to as part of such accounts, though ultimately their emphasis on the challenges posed by social media to young people's control over the information they share tends more towards an explanation of such online environments as uniquely digital.

This article reflects on use of the teenage bedroom analogy as means to explore broader questions about the role of social network sites in young people's lives and, in particular, the extent to which they provide environments sufficiently personal, secure and under control to enable productive forms of socialisation, performance and identity work. I begin by considering early discussions of the teenage bedroom as a way to understand social network sites, identifying some difficulties with the metaphor in light of recent developments in the affordances and uses of such media. I go onto explore conceptualisations of social network sites as public space and discussions of the challenges to privacy faced by young people in such environments. Such accounts, I suggest, question the continuing value, not only of the bedroom analogy, but of any face-to-face spatial comparators, on the basis that the affordances of social media render them unique social environments with respect to young people's ability to control what they share with whom.

For all their value I suggest such accounts may under-estimate the control many young social media users retain over their communications, control that I suggest may not be 
entirely dissimilar to the interactive strategies that operate in offline environments. While this may not, in itself, warrant a full reprieve for the bedroom analogy, I underline how the latter may nevertheless help us to grasp the individual-centredness of social network sites, with respect to connections, content and feel. More specifically, I suggest that it might enable us to attend in greater detail to the ways such sites function as vital forms of personal home territory for young people whose daily lives traverse a range of contexts.

\section{Virtual Bedrooms?}

Use of the teenage bedroom as a means to understand online spaces began in the 1990s in the era of the personal homepage. Scholars such as Chandler and Roberts-Young (1998) and Walker (2000) suggested that, even though they were publicly accessible, the way such sites were centred on and controlled by a particular individual replicated the status of the bedroom as the primary individual-centred physical territory in many young people's lives. Such studies emphasised, in particular, how the performance of identity through personal interests, tastes, likes and friendships approximated the display of identity visible on teenage bedroom walls. Such studies also provided an early glimpse of the ways Erving Goffman's understandings of social performativity might be applied to the exhibition of identities in such spaces.

Moving the bedroom analogy into the era of social media, Hodkinson and Lincoln (2008) explore the apparent similarities between young people's experience of bedroom spaces and their use of interactive online journals on the LiveJournal platform, an early blog-based 
social networking site. The discussion explores how LiveJournal users favourably contrasted the sense of personal territory, intimacy and individual control afforded by the platform with their prior experiences of public online discussion forums. Personally owned space was equated with greater scope for free, safe and intimate self-expression. One respondent said, 'It's about ownership of space. My LiveJournal is my space, I own it... I think a lot less about [what I write in my] LiveJournal because LiveJournal is safe space. It's my space, it's safe space' (Hodkinson and Lincoln, 2008: 32). Consistent with this, an ethic of respect for the personal space of each user and their right to use it as they please was in evidence, enabling users to feel released from collective expectations and able to blog about the minutiae of everyday personal life as well as debates and controversies. And crucially, users invested confidence in the ability to restrict access by rendering their journal friends-only. A shared symbolic understanding of journals as personal territory was combined, then, with practical control over who could enter one's space.

LiveJournal users also invited comparisons with the teenage bedroom, argue through their marking out of online territory through customised formats and the creation of personal profile pages, something one respondent compared explicitly to having her own room: 'it's like being given a room - and you can furnish it the way you want and paint it the way you want - and you make it your room' (ibid.: 32). As in the case of earlier homepages, argue Hodkinson and Lincoln, such displays contribute to the establishment of journals as intimate personal space, while performing a similar role to bedroom walls with respect to the reflexive performance and mapping of identity. The establishment and periodic updating of such identity displays within personal space are deemed integral to young people's attempts to construct meaningful identity narratives out of their fast 
changing adolescent lives. The use of interactive journals, then, is argued to carry similar potential to the bedroom in affording particular forms of identity performance alongside a reflexive mapping out and making sense of youthful transitions and biographies (Giddens, 1991; Denora, 2000).

Unlike personal homepages, however, the fixed display of identity provided by profile and decoration forms merely the backdrop to a plethora of ongoing updates and interactions that are embedded in everyday events and emotions. Such ongoing conversation comprises a more immediate and collaborative form of identity work than the decoration of the space itself (see Lincoln and Robards, 2014). As well as acting as a form of personal media, as in a diary (Lindemann, 2005), scrapbook (Good, 2013) or exhibition (Hogan, 2010), then, Hodkinson and Lincoln's analysis invites an understanding of LiveJournal as personal space in which young people spend time with trusted others on terms of their own choosing, making sense of their place in the world as they converse.

\section{Bedrooms or Publics?}

While various scholars have adapted the bedroom analogy as a means to make sense of elements of more recent social network sites (Pearson, 2009; Robards, 2010; Downs, 2011; Lincoln, 2012; Lincoln and Robards, 2014), it nevertheless has a number of limitations, some of which have become accentuated in light of changes to the affordances of social media as such platforms have evolved. 
Most obviously, the size of the potential pool of fellow interactants on most social network sites is, typically, on a different scale than could be accommodated by the bedroom - and studies have indicated that, while such platforms can support or enhance close friendships, their affordances sometimes are suited to communication with a wider number of superficial acquaintances, or 'weak ties' (Ellison et al, 2007). Not only are individuals apparently conversing with many people, then, but with individuals whose superficial relationship with one another appears at odds with the intimacy evoked by the bedroom analogy. Depending on the ubiquity and orientation of the site in question, friend- or follower-lists also can bring together people from different spheres of life, something made particularly likely on Facebook by its insistence on real names (Lincoln and Robards, 2014). As we shall see, social network platforms differ in their precise affordances as well as being open to a variety of different sorts of use. In many cases, though, they would seem to comprise spaces of communication less restricted, narrow and intimate than the teenage bedroom analogy implies (Robards, 2012; 2014).

Another development that sits uneasily with the bedroom analogy is a reduction in the importance of stable, holistic displays of identity through the customised profiles in favour of more immediate forms of interaction (Lincoln, 2012). In contrast to the extensive emphasis on the decoration of personal space in the heyday of MySpace, the onset of Twitter, the evolution of Facebook and the development recent platforms such as SnapChat, illustrate greater emphasis on fleeting, ephemeral forms of everyday interaction (Velez, 2014). While significant personal customisation of the appearance of one's online space remains a key feature of some contemporary platforms - notably TumbIr (Renniger, 2014; 
Tiidenberg, 2015) - such fixed set-piece displays appear to be of less importance to most young people's social network use today than communication in the moment, something exacerbated by cultures of 'always on' access via mobile technologies. Kaun and Stiernstedt (2014), for example, suggest in relation to Facebook, that 'users' temporal experience is one of immediacy, ephemerality, "liveness" and flow... for the user, the experience and feel... is one of rapid change...'. While such conversation forms part of Hodkinson and Lincoln's analysis, the extent to which it has eclipsed more carefully curated identity exhibitions (Hogan, 2010) on many platforms raises questions about whether comparisons with teenage bedroom walls retain purchase. Recent respondents of Lincoln (2012), for example, noted a loss of emphasis on customised design of space in favour of ongoing conversation with friends in their migration from MySpace to Facebook, noting that the former had greater similarities with use of their bedroom than the latter.

The increasing centrality of newsfeeds, or equivalent, to many social network sites, raises further questions about the bedroom analogy. Enabling individuals to communicate simultaneously with numerous peers, the newsfeed acts as the primary conduit for communication. Rather than visiting and spending time in one another's discrete spaces, individuals may instead be considered to be spending time in the liminal 'space' of their personalised news feed, where they view and interact with an aggregated set of communications from a range of friends, acquaintances or groups. And depending on the platform, this may enable a wider range of communication than in earlier social media, including, in the case of Facebook, status updates, photo tagging, comments, group posts, personal messaging and so on. Within or across sites, users may find themselves 
communicating simultaneously to different audiences, something inadequately captured by the notion of spending discrete blocks of time with a particular set of others in intimate spaces.

Perhaps such factors corroborate danah boyd's $(2007 ; 2014)$ understanding of social network sites as primarily public spaces of interaction. Boyd's careful analysis, centred on a series of meticulous research projects with young users, recognises a complex blurring of private-public boundaries. Yet, ultimately, young people's motivation to spend time on social network sites is understood to reflect their desire 'to gather en mass with friends, acquaintances, classmates and other teens' (boyd, 2014: 22, my emphasis). Boyd notes that the appeal of social media may reflect ever-greater restrictions on young people's access to public physical spaces during their leisure time, whether through their prohibition from hanging out in privatised shopping malls, increasing regulation of their occupation of outside spaces or the broader tendency for parents to keep teen children indoors as much as possible. Social network sites, boyd argues, have become 'the only "public" spaces in which teens can easily congregate with large groups of their peers' (ibid.: 21 , my emphasis). And, consistent with this, boyd uses public spatial analogies for the 'networked publics' that characterise social network site communication:

In some senses mediated publics are similar to the unmediated publics with which most people are familiar - parks, malls, parking lots, cafes etc. Teens show up in order to connect with their friends. Other people might be present and might be brought into the circle of conversation if they're interesting, or ignored if not (2007: 2). 
Through conceiving of young people as coming together within public space, then, boyd's analysis largely rejects the notion that they are conversing in personal, private environments. In support, boyd devotes considerable attention to young people's comparative lack of control, as she sees it, over access to the conversations, images and artefacts they share. Directly responding to Hodkinson and Lincoln's conception of social network sites as personal, bedroom-like space, she suggests that, "because of the properties of social media, creating boundaries around these online spaces is far more difficult' (2014: 47).

\section{Context collapse and loss of control}

One of the primary points established across boyd's work is that, while they share certain features with the park and the mall, 'networked publics' differ substantively from physical public spaces with respect to how difficult it is for young people to control access to themselves and their content. Contrary to popular stereotypes, boyd shows, young people invariably are concerned about social privacy (though less about possible access to their communications by distant corporations or governments) and often seek to protect it. The problem is that the affordances of social media severely compromise their ability to do so. In particular, the permanence of communication and ease with which content can be retrieved or replicated are emphasised, alongside a broader contention that, as a result, social media are characterised by 'invisible audiences'. Content or conversation intended for a small group of friends, then, may end up being accessed by limitless others (boyd, 2007; 2014). 
Alongside others, boyd understands such a possibility through the notion of context collapse. As per Goffman's dramaturgical understanding of face-to-face interactive behaviour (1956; 1957), the 'performances' given by individuals online often are crafted for particular audiences - and understanding of one's audience is essential to effective performance. Unlike in public physical spaces, however, it is argued that on social network sites the boundaries between otherwise discrete social groups - that normally would enable what Goffman calls 'audience segregation' - are insecure, resulting either in an inability to perform or a damaging mismatch between intended and actual audience. Thus, the permanence and searchability of online content, the possibility of being watched while typing, the potential for friends to copy and paste comments and a range of other factors may render potentially compromising revelations, photographs or comments intended for peers accessible to wider groups of young people or even to parents, teachers, college admissions staff or employers (boyd, 2007; 2014; also see Livingstone, 2008). In physical public space, boyd argues, a group of friends will adapt to the presence of approaching outsiders but, on social network sites, it is impossible to know who is present at the time or who may view the interaction out of context long after it took place. As a Facebook user in a study by Raynes-Goldie (2010) pointed out, 'Facebook makes things that should just have happened in passing totally permanent and public'.

The collaborative, social nature of content sharing is argued to create particular challenges to young people's control over privacy (Lincoln and Robards, 2014; Marwick and boyd, 2014). Photographs may be posted and tagged by friends resulting in them 
being viewed and shared by a range of audiences, comments to friends' posts are broadcast to the posters' friends-list or beyond, a friends' comment on an old photograph may cause it to appear at the top of friends' news feeds. Various studies have indicated how such complexities might work to young people's disadvantage, not least those centred upon the displays groups of young people sometimes make of themselves before, during and after intoxicated nights out. Goodwin and colleagues (2014), for example, outline how, notwithstanding the importance of the exchange of online 'drunken narratives' as part of young people's social bonding, there is a considerable risk that such accounts may end up being accessed far beyond those they were intended for, with potentially harmful consequences:

there is a potential loss of control over the context in which such texts are read, re-read and shared (potentially ad infinitum). The meanings subsequently made of the behaviour, possibly years after the fact, can be radically different from those of the user's peer group, and the potential consequences not always positive... The threat posed by such forms of "context collapse" is particularly heightened for youth pursuing online activities that sit outside dominant social norms... (Goodwin et al, 2014: 62)

While Goodwin and colleagues note that the 'paradigmatic example' of context collapse might be a google-search by an eavesdropping outsider, such as an employer, the problem also pertains to the range of people located within an individual's network, especially on platforms with broad usage profiles such as Facebook. Often bringing together individuals from different contexts - college friends, siblings, old school friends, work colleagues and others - such a diverse audience may make it difficult for individuals 
to grasp the context in which they are operating and/or to present an effective, coherent and nuanced impression of themselves. Boyd (2014) describes the difficulties one user had with the tendency of his sister and cousin to comment on Facebook posts intended for his college friends, something that adversely affected his interactions with the latter. Alternatively, individuals may lose sight of the breadth of their networks, as in a respondent of Robards' (2014) who posted a series of intimate messages to his girlfriend only to find himself confronted with a print-out of the exchanges at work as an office joke.

For boyd, then, young people's attempts to establish themselves socially and make sense of their place in the world are taking place in a perilous environment in which control over the reach of what they share is an ongoing battle. Social worlds regularly 'collide uncontrollably' (2014: 36) in online spaces where sharing publicly becomes established as a default approach, the achievement of greater privacy requiring extensive vigilance (Raynes-Goldie, 2010). Such a situation, argues boyd, inverts the norms of socialisation in physical spaces, whereby interactions are usually restricted to small, visible groups. Importantly, such an analysis not only takes us beyond the aforementioned bedroom analogy but indicates that online interactive environments are fundamentally distinct from any form of physical space. Thus, Goodwin et al conclude that, within such an environment, 'the line between crafting an authentic and autonomous online subjectivity and of losing control of the management of the self entirely, becomes very thin indeed' (2014: 70). Similarly, for boyd, 'teens are struggling to make sense of who they are and how they fit into society in an environment in which contexts are networked and 
collapsed, audiences are invisible and anything they say or do can easily be taken out of context' (2014: 53).

\section{Access control and privacy strategies}

While offering carefully researched challenges to moral panics relating to youth naivety or nonchalance with respect to privacy, such discussions usefully outline the challenges that may face those who spend time on such platforms. Greater scrutiny may be warranted, however, when it comes to the level of fit between the extent of the loss of control inferred in some such accounts and the everyday realities of most young social media users.

Partly, this is because such accounts have a tendency to be unclear whether the negative scenarios outlined do or do not apply to users who use friends-only or comparable access controls. Many would probably agree that, if identifying or identifiable content is posted publicly, without the use of any form of access control, young people may indeed find themselves vulnerable to snooping by uninvited audiences or uncontrolled sharing of personal content beyond their immediate networks. Further attention might be devoted to whether or not such breaches are as common or costly as is sometimes implied and the circumstances in which they take place, but considerable possibilities and risks present themselves. But what if friends-only or equivalent controls are available and switched on? 
Evidence suggests that, partly as a result of a gradually increasing awareness of potentially damaging threats to privacy, the proportion of young people who use friendsonly controls on sites like Facebook is increasing. While in 2008 , Lewis et al found that only $33 \%$ of teenagers used privacy controls, the Pew Internet Research Project reports that, by 2012 , only $14 \%$ of Facebook users had fully public profiles, with $60 \%$ fully 'friends-only' and $25 \%$ restricted to 'friends of friends'. The survey also revealed high confidence among teens in the use of such controls, with only $9 \%$ finding them somewhat or very difficult to use (Madden et al, 2013). In a crucial subsequent development, Facebook changed the default setting for all new accounts to friends-only as well as simplifying and publicising its privacy controls (Gross, CNN 2014). The move is widely seen as a response to the increasing popularity among young people of newer platforms such as Snapchat that centre on the provision of private, secure channels for communication between limited groups of friends (Velez, 2014). Without wishing to underestimate the significance of those whose content remains fully-public - including users of platforms such as Twitter that do not offer friends-only controls - there may be a direction of travel among teens towards friends-only communication as default where it is available.

Of course, restricting communication to one's immediate network does not provide insulation from all the difficulties outlined. Depending on the platform being used and their approach to friending, individuals may still be communicating to a diverse network that collapses different social spheres. $9 \%$ of young people in a recent European study indicated they accept all friend requests, for example (Livingstone, 2015) while US research on Facebook indicates that, even for the more selective majority, friends-lists 
frequently include family members and, more occasionally, teachers or coaches, even if they remain dominated by friends and acquaintances of the same age (Madden et al, 2013). And only a minority in the latter study indicated they used custom lists to filter who could see what; for $81 \%$ of the sample, all friends had access to everything they posted (ibid.). Even with friends-only controls on, then, young people may be interacting simultaneously with a broad group of people, with implications for the kinds of selfperformance, bonding or reflexive identity-work that may be possible without loss of face.

Yet, it is less easy to support the contention, in this scenario, that contexts are collapsing uncontrollably or that there is a particularly acute problem with invisible audiences. Friend-lists may be broad on platforms such as Facebook, but even here they consist of a hand-picked and relatively consistent sets of individuals, each of whose presence has explicitly been consented to. And many young people periodically review their friendslists, reminding themselves of who is present and removing those with whom they no longer wish to interact (Madden et al, 2013). Young people with 'friends-only' profiles may sometimes feel restricted or awkward about the simultaneous presence of people from different contexts, but implications that they have little idea of who can see or interact with the content they share or that they are especially vulnerable to intrusion by people outside their network are more questionable.

Recent research by Robards and Bennett (2012) suggests that, in spite of their capacity to incorporate diversity, friends-lists tend to gradually stabilise as people move through adolescence, coming to form a relatively predictable and consistent part of individuals' 
communicative spheres. In this understanding, although it may bring together contexts that otherwise would be separate, the friends-list crystalises into a network of friends and acquaintances with whom an individual comes to feel 'at home', a point to which we shall return later (ibid.). In such a circumstance, it seems plausible that, as their familiarity with the particular make-up of their personal network rises, individuals are liable to learn to perform and converse effectively in an environment characterised by a degree of familiarity, stability and, even, predictability.

There remain a variety of ways in which information may get into the wrong hands, of course. Individuals may occasionally slip-up through failing to take into account all those present, may comment on a friend's post without knowing that person's friending policy (Marwick and boyd, 2014). They may find their content or images shared, reproduced or tagged by friends (Raynes-Goldie, 2010) or that information from the past suddenly reappears (Robards, 2014). Further scrutiny may be warranted, however, when it comes to the frequency of such breaches and the extent of the damage caused. Are most young people experiencing damaging instances of loss of control on a daily basis or do their everyday online interactions - for all their complexities - tend to proceed a little more smoothly than this most of the time? A partial indication only is provided by recent survey research by Madden et al (2013) in which only $4 \%$ of teens said that they had shared something sensitive online that had subsequently caused problems for them or their family.

One thing becoming clear is that, in addition to their use of direct access controls, young people are becoming adept in the use of a range of other strategies through which to 
negotiate the privacy challenges they may face in online social environments (Lange, 2008; Livingstone, 2008; Raynes-Goldie, 2010; Lincoln and Robards, 2014; Light and Cassidy, 2014; boyd, 2014; Marwick and boyd, 2014). Light and Cassidy (2014) draw attention to an array of 'disconnective practices' utilised by users to establish control over what they communicate to whom, including declining invitations, defriending people, detagging themselves from content and deleting posts or images from their timelines. Rather than being especially subversive, Light and Cassidy regard such practices as an essential and everyday part of Facebook culture. Another important strategy on those sites that afford it is to develop accounts under usernames distinct from one's real name and carefully control who knows about them. On Tumblr this contributes to a tendency among some users to experience their communication as less inhibited than on Facebook, in spite of the tendency for content to be publicly available (Renninger, 2014). Tumblr users also use a range of other strategies to protect their privacy, from directly confronting unwelcome strangers who breach privacy to deliberately posting poor quality selfies to discourage the reblogging of content outside limited circles of friends (Tiidenberg, 2015).

Marwick and boyd (2014) detail a range of privacy strategies across different social media platforms, including the selective use of different platforms as a means to re-segregate audiences and the informal encoding of communication so that meaning is discernable only by a limited group. Although their account affords considerable agency and ingenuity to their participants, the achievement of privacy is inferred as an ongoing struggle 'in a networked ecosystem in which contexts regularly blur and collapse' (2014: 1063). One is still left with the impression of young people valiantly swimming against 
the tide in a uniquely hazardous environment. Without underestimating the unique challenges that social network sites can pose, it may be worth asking whether, in their normal everyday experience, most young people find their interactions in such environments to be quite such an uphill struggle as compared, for example, to their negotiations of privacy in offline spaces. It is worth remembering that studies continue to indicate that most young people feel positive overall about their experience of social network sites. Madden et al (2013) found, for example, that 'in broad measures of online experience, teens are considerably more likely to report positive experiences than negative ones'. We should, of course, be cautious in our interpretation of such findings and more detailed comparative research of young people's off and online experiences of social life would be of great value in illuminating the situation further. That the affordances of online media have implications for privacy is clear, but the extent to which such differences render negotiations over privacy an entirely different game from those played out in other spaces remains unclear, I would suggest.

Marwick and boyd's analysis of young people's privacy strategies usefully highlights that, rather than relying entirely on technical solutions, or isolated individual control, the achievement of 'networked privacy' revolves around the establishment of collaborative, social understandings and shared sets of informal values and ethics among networked peers: 'no technical solution can provide complete reassurance. Instead, teenagers often rely on interpersonal relationship management to negotiate who shares what about them, who does what with their information, and how their reputations are treated (2014: 1061). The point is astute and bears resemblance to findings elsewhere - including Davis' (2014) emphasis on the role of shared peer understandings to the achievement of 
effective and secure online interactions and Tiidenberg's (2015) discussion of the maintenance of shared ethics and trust among like-minded groups of Tumblr users.

But such observations draw as much attention to similarities in the negotiation of privacy between on and offline contexts, as they do to differences. After all, in Goffman's accounts of face-to-face interaction, the presence of mutual interests, collaboration and shared understandings in the validation of performance is essential to the avoidance of loss-of-face, including through breaches of context (1956; 1957). The precise ways privacy might be breached or loss-of- face suffered in online social environments may be different from those in physical spaces, and the prospect of precise replication and scale of potential sharing may sometimes render breaches especially damaging. But the need to carefully negotiate social performance with and between audiences from different contexts is not, in itself a phenomenon unique to social media and neither is young people's reliance on and trust in those with whom they interact in order to be able to communicate securely.

Taken together, then, the increasing use of tools that restrict communication to a handpicked immediate network of fellow-interactants and the everyday deployment of a range of informal strategies to restrict and filter what is shared with whom may indicate greater levels of control among young people over their online interactions than is sometimes supposed. And there may be good reason to further explore through detailed research, points of comparability between young people's negotiation of the interactional opportunities and challenges in online social network spaces and their approaches to such 
issues in other sorts of environment, including physical space. At which point, I return to the subject of the usefulness of the particular spatial metaphor of the teenage bedroom.

\section{Personal 'home territories'}

Notwithstanding the kinds of communicative control I have alluded to above, the bedroom itself remains an awkward fit with respect to the form taken by contemporary social networking site environments. As already noted, the analogy jars with the prominence of the newsfeed, the relative decline in the importance of space/profile customisation, the complexity of different formats and scales of communication and the breadth of the networks with whom individuals often communicate.

We might also note that, in a broader sense, the analogy has tended to afford a somewhat individualistic way of looking at young people's identities in particular spaces (Robards and Bennett, 2012). Emphasis on the significance of personal space for the disembedded, multi-affiliated individual negotiating identity and transitions in a fluid, uncertain world (Hodkinson and Lincoln, 2008; Robards and Bennett, 2012) may, after all, only offer part of the story with respect to young people's online sociability. Because the analysis centres on the individual and individual-space, it is not particularly well-placed to grasp, for example, the continuing significance of structural allegiances and constraints, the ways discernable collective groups or subcultures play themselves out via online networks (e.g. Williams, 2006; Baym, 2007; Hodkinson, 2007), or the importance of such 
communities in influencing patterns of identity rather than merely as resources for individualised identity-making. Crucially, studies of the ways collective youth cultures manifest themselves online tend to illustrate that, rather than being confined anywhere in particular, they invariably traverse a multitude of networked spaces, both public and private.

Yet, if it can be combined with understanding of the multi-spatial character of what Baym (2007) calls 'networked collectivism' and attentive to structural patterns, the examination of individual-centred identity-work and socialisation in particular spaces retains considerable value, not least in developing a more nuanced understanding of the interplay between collective and individual identity for differently located young people and of the relationship between young people's liminal position across territories and the extensive time they spend within particular spaces.

For all the deficiencies identified, I want to suggest that the bedroom analogy may yet have some value as part of this, through helping us make sense of the way social network sites can function as what we might call familiar, personalised home territories for young people, amidst complex, multi-spatial lives.

Recent adaptations of the bedroom analogy have shown how a spatial metaphor need not be a perfect fit in order to help us make sense of some of the key affordances and uses of digital environments. Pearson's notion of the 'glass bedroom' captures the way in which, in spite of the size or immediate networks who may potentially access what an individual shares, young people's everyday use of social media is liable to be dominated, 
much of the time, by conversations with smaller groups (2009). Such conversations may be observed by larger numbers of passing acquaintances, or sometimes strangers - and these larger groups may be interacted with more directly from time to time - but the prevalence of communication between close friends suggests the space may, after all, retain a degree of the personal intimacy captured by the bedroom analogy, at least some of the time:

Inside the bedroom, private conversations and intimate exchanges occur, each with varying awareness of distant friends and strangers moving past transparent walls that separate groups from more deliberate and constructed 'outside' displays. Some exchanges are constructed with an awareness of the users beyond the glass walls and play to them, exchanging information and ideas using common signs and symbols. Users outside the bedroom may engage or not; they may move on, or they may find themselves invited into the bedroom to continue a given conversation. Other exchanges are more closely guarded, with users huddled together, ignoring those outside, and expecting those outsiders not to stand and watch... (Pearson, 2009)

While Pearson is clear that intimate conversations on social network sites do not amount to any sort of 'back stage' (also Hogan, 2010), her retention of the bedroom concept indicates they do retain certain features familiar to private communication. Pearson's observation is important and, in addition to the obvious point that closer friends tend to take a greater interest in one another's content, the propensity for communication to be dominated by smaller groups of friends, even in the case of broad friends-lists, is surely set to become more concentrated by the ongoing development of newsfeed algorithms that filter and prioritise content on the basis of previous patterns of interaction. And the rapid growth of 
newer platforms explicitly oriented to intimate conversation with smaller groups of friends may further reinforce the point. Velez (2014) points out, for example, that Snapchat interactions tend to involve groups of friends considerably smaller than most Facebook friends-lists and that, together with the ephemerality of content on which the platform centres, this leads such conversations to have a particularly intimate feel. Meanwhile, Tumblr blogs, although often publicly accessible, are, according to Tiidenberg's (2015) study of NSFW content, frequently regarded by users as 'safe spaces of self-expression' whereby interaction is oriented to relatively discrete and limited sets of trusted followers known to Tiidenberg's respondents as 'my Tumblr people'. The similarity of such 'safe space' rhetoric to the accounts of Hodkinson and Lincoln (2008) in their earlier studies of LiveJournal are striking.

Meanwhile, drawing on Pearson's work on the glass bedroom amongst others, Lincoln and Robards (2014) recently have utilised the bedroom analogy a means to frame their ongoing explorations of young people's display and mapping of identity on Facebook. Drawing direct comparisons between the strategies of identity display and control in the bedroom and on Facebook, the account identifies differences as well as recognising diversity in the uses of both spaces by differently located individuals, not least with respect to disagreement between respondents on whether Facebook or indeed the bedroom itself, functions as a form of curated identity display. Yet the comparison also shows clear points of comparability with respect to young people's symbolic expressions of identity and practical strategies of control within the two environments. 
Most recently, Lincoln and Robards' research has focused on the significance of the Facebook timeline and the ways their respondents are utilising increased possibilities to engage with an easily searchable archive of activity (see Robards, 2014). The importance of fixed, customisable profiles may have receded, they suggest, but engagement with the timeline, through searching, examining, reminiscing, editing and shaping, enables forms of reflexive identity performance and mapping comparable to those envisaged on earlier platforms. In this sense, the timeline affords, they argue, a renewed emphasis on permanent establishment of identity and biography that works alongside more fleeting, everyday forms of communication and identity work in the moment. In a manner only partially distinct from the bedroom, the ways young people are utilising the timeline, argue Lincoln and Robards, enables a process of mapping and making sense, a reflexive construction and reconstruction of growing up biographies. Notwithstanding the divergence between platforms with respect to the issue of archiving (Snapchat's popularity reflecting its emphasis on immediate and ephemeral forms of interaction and identity work, for example, while various other platforms continue to feature archived, dated entries), the study offers a reminder of how sites that utilise accessible personal achieves continue to afford extensive long-term identity mapping.

Drawing Pearson and Robards and Lincoln's insights about the continuing potential value of the bedroom comparison together with my earlier discussion of questions of individual privacy and control, I want to suggest that social network sites have a tendency to act as personal home territories for young people, as they negotiate the development of identities, trajectories and affiliations as they grow older in complex, multi-spatial environments. Numbers of fellow-interactants may be high, intimate interactions may 
sometimes be viewable by others and personal control may be subject to challenges but, through the ability to limit most communication to personalised networks through access controls, choice of usernames and a range of other connective and disconnective strategies, such personal control may be greater than in the various other spaces young people traverse. As a consequence, individuals may indeed often come to feel broadly 'at home' within their social network environments and with the regular fellow interactants therein, as Robards and Bennett's (2012) research suggests. Meanwhile, an individualorientation in terms of what people share and who they converse with offers the potential for different facets of individual identity, lived out through a range of other spaces, to be brought together, expressed and, in so doing, mapped and made sense of. Perhaps the highly complex de-centred, fragmented post-modern selves described two decades ago by the likes of Turkle (1995) may be, at least partly, stabilised and made more comprehensible through ongoing conversation, exhibition and reflexive identitywork on familiar, personal home territories.

There are, of course, complexities to consider. For some users, much of their online interactive and identity work will take place via a single account on one platform while in other cases, personal territories will be established and utilised in two, three or more online social environments. And, as we have seen, the precise affordances of these platforms will vary, offering the prospect of territories with different sets of orientations, opportunities and challenges. Yet they continue to share, amongst other things, an orientation to the individual as the primary focal point on which the sharing of content via networks of interaction are organised. For all of their distinct affordances, they - like the teenage bedroom - comprise environments which, from the point of view of each 
user, are centred on and organised around themselves and can be used as familiar base points for broader patterns of socialisation and identity. In spite of its failings as an analogy, then, the bedroom, which for many young people in late capitalist societies comprises the only physical space that performs this sort of a role, may come closer to helping us appreciate the potential significance of social network sites as individualcentred base points than do public metaphors such as parks, malls and the like.

Thinking about some of the roles of the bedroom for young people with respect to interaction and identity, then, may continue to be helpful in the quest to make sense of the specific importance of personal-oriented online space as a focus for young identity work and of its connections to other forms of space both on and offline. And whilst important in the case of those young people who also have the benefit of physical bedroom space of their own (Lincoln, 2012), the analogy may also prove interesting as a way of thinking through whether social network sites can function as personal identity spaces for those whose disadvantaged social position or circumstances mean they do not have access to discrete physical space of their own (see Neary 2015). In so doing, it may address the criticism that research on teenage bedrooms as identity spaces has tended to be restricted to the practices and identities of middle-class youth.

\section{Conclusion}

Against the context of increasingly compulsory and always-on cultures of social media use among young people in late capitalist societies, this article has dwelt upon the 
teenage bedroom analogy as a means to make sense of some of the affordances and uses of social network sites with respect to young people's social lives and identities. Having summarised earlier uses of the analogy as a means to capture young people's experience of social media as a personal social and identity space, I went on to explore possible difficulties with it. I dwelt, in particular, on narratives about young people's loss of control over privacy in contemporary social media environments that collapse contexts and undermine boundary control. I argued that, in spite of the specific challenges young people face, it is worth asking whether proclamations of uncontrollably collapsing contexts and invisible audiences fit with the everyday realities of most young people's interaction in such environments. For most people, most of the time, I asked, might the everyday experience of communicating via social media feel a little more under control? We might also revisit, I suggested, the extent to which similarities remain between young people's privacy experiences and strategies online as compared to their negotiation of such issues in physical spaces. Detailed comparative research may help us to answer such questions.

Returning to the teenage bedroom analogy, I drew on recent adaptations in order to show that, in spite of being an imperfect fit, the metaphor may still enable us to capture some key affordances and uses of such environments that are obscured by public spatial analogies. I dwelt on the notion that, in addition to retaining an ability to restrict most communication to limited, familiar networks, individuals may find that their everyday conversation remains dominated by smaller groups of close friends - and that such apparent intimacy may yet enable young people to take advantage of the individualorientation of social network sites to exhibit, play out and reflexively map identities and 
transitions. And I argued that, in moving the discussion forwards, we might usefully conceive of social network sites as performing the role of personal home territories for young users - familiar, individually-centred base-points for sociality and identity in the context of complex, multi-spatial lives and identities.

Of course, such a formulation by no means captures all we need to understand about social network sites or indeed of the broader establishment and living out of young identities online. Rather, it invites a particular angle of study, centred on the use of particular familiar person-centred environments by individuals in the construction, making sense and playing out of identity. Amongst other things, such an emphasis can be combined and integrated, in future research agendas, with greater understanding of the traversing by individuals - and of the communities to which they attach themselves - of multiple networked spaces.

\section{References}

Abbott-Chapman J. and Robertson M. (2001) 'Youth, Leisure and Home: Space, Place and Identity', Leisure and Society 24 (2): 485-506.

Baym N. (2007) 'The New Shape of Online Community: The Example of Swedish Independent Music Fandom', First Monday 12 (8).

boyd d. (2007) 'Why Youth (Heart) Social Network Sites: The Role of Networked Publics in Buckingham, D. (ed.) Youth, Identity, and Digital Media, Cambridge, MA: MIT Press.

boyd d. (2014) It's Complicated: The Social Lives of Networked Teens, Yale University Press. Chandler D. and Roberts-Young, D. (1998) 'The Construction of Identity in the Personal Homepages of Adolescents', URL: http://www.aber.ac.uk/media/Documents/short/Strasbourg.html

Davis K. (2014) 'Youth Identities in a Digital Age: The Anchoring Role of Friends in Young People's Approaches to Online Identity Expression' in Bennett A. and Robards B. (eds.) 
Mediated Youth Cultures: The Internet, Belonging and New Cultural Configurations, London: Palgrave.

DeNora T. (2000) Music and Everyday Life, Cambridge: Cambridge University Press

Downs, C. (2011) 'Playing in a Virtual Bedroom: youth leisure in the Facebook generation', in Jeanes R. and Magee J. (eds.), Children, Youth and Leisure, Leisure Studies Association, 1531.

Ellison N., Steinfield C. and Lampe C. (2007) 'The Benefits of Facebook "Friends:" Social Capital and College Students' Use of Online Social Network Sites', Journal of Computer Mediated Communication, 12: 1143-1168.

Giddens A. (1991) Modernity and Self-Identity: Self and Society in the Late Modern Age, Cambridge: Polity.

Goffman E. (1956) The Presentation of Self in Everyday Life, University of Edinburgh.

Goffman E. (1967) Interaction Ritual: Essays in Face-to-Face Behaviour, Chicago: Aldine Publishing Company.

Good K. D. (2013) 'From scrapbook to Facebook: A history of personal media assemblage and archives', New Media and Society 15 (4): 557-73.

Goodwin I., Lyons A., Griffin C. and McCreanor T. (2014) 'Ending Up Online: Interrogating Mediated Youth Drinking Cultures', in Bennett A. and Robards B. (eds.) Mediated Youth Cultures: The Internet, Belonging and New Cultural Configurations, London: Palgrave.

Gross D. 'Facebook Privacy now Defaults to Friends Only', CNN, URL: http://edition.cnn.com/2014/05/22/tech/social-media/facebook-privacy/ Hodkinson P. (2007) 'Interactive Online Journals and Individualization', New Media and Society, 9 (4): 625-50.

Hodkinson P. and Lincoln S. (2008) 'Online Journals as Virtual Bedrooms: Young People, Identity and Personal Space', YOUNG 16 (1): 27-46.

Hogan B. (2010) 'The presentation of self in the age of social media: Distinguishing performances and exhibitions online', Bulletin of Science, Technology and Society 30 (6): 377-386.

Kaun A. and Stiernstedt F. (2014), 'Facebook time: Technological and institutional affordances for media memories', New Media and Society 16 (7): 1154-1168

Lange P. (2008) 'Publicly Private and Privately Public: Social Networking on YouTube', Journal of Computer Mediated Communication 13: 361-380

Light B. and Cassidy E. (2014) 'Strategies for the suspension and prevention of connection: Rendering disconnection as socioeconomic lubricant with Facebook', New Media and Society, 16 (7): 1169-1184. 
Lincoln S. (2012) Youth and Private Space, London: Palgrave.

Lincoln S. and Robards B. (2014), 'Being strategic and taking control: Bedrooms, social network sites and the narratives of growing up', New Media and Society, online before print DOI: $10.1177 / 1461444814554065$.

Lindemann K. (2005) 'Lives Online: Narrative Performance, Presence and Community in LiveJournal.com', Text and Performance Quarterly 25 (4), pp. 354-372.

Livingstone S. (2008) 'Taking risky opportunities in youthful content creation: teenagers' use of social network sites for intimacy, privacy and self-expression', New Media and Society 10 (3): 393-411.

Madden, M., Lenhart, A., Cortesi, S., Gasser, U., Duggan, M., Smith, A. and Beaton, M. (2013) 'Social Media, Teens and Privacy', Pew Internet Research Project Report, URL: http://www.pewinternet.org/2013/05/21/teens-social-media-and-privacy/

Marwick A. and Boyd D. (2014) 'Networked privacy: How teenagers negotiate context in social media', New Media and Society 16, 7: 1051-1067.

Neary J. (2015) Changing contexts: young people's experiences of growing up in regeneration areas of Glasgow. Phd Thesis. University of Glasgow: UK

Pearson E. (2009) 'All the wide web's a stage: the performance of identity in online social networks', First Monday 14 (3).

Raynes-Goldie K. (2010) 'Aliases, Creeping and Wall-Cleaning: Understanding Privacy in the Age of Facebook', First Monday 15 (1).

Renninger B. (2014) "Where I can be myself ... where I can speak my mind" : Networked counterpublics in a polymedia environment', New Media and Society, online before print, DOI: $10.1177 / 1461444814530095$.

Robards B. (2010) 'Randoms in my bedroom: Negotiating privacy and unsolicited contact on social network sites, PRism 7 (3): http://www.prismjournal.org

Robards B. (2014) 'Mediating Experiences of "Growing Up" on Facebook's Timeline: Privacy, Ephemerality and the Reflexive Project of Self', in Bennett, A. and Robards, B. (eds.) Mediated Youth Cultures: The Internet, Belonging and New Cultural Configurations, London: Palgrave.

Robards B. and Bennett A. (2012) 'Mytribe: Post-Subcultural Manifestations of Belonging on Social Network Sites', Sociology 45 (2): 305-17.

Tiidenberg K. (2015) 'Boundaries and conflict in a NSFW community on Tumblr: The meanings and uses of selfies', New Media and Society, online before print, DOI: DOI: 10.1177/1461444814567984. 
Turkle S. (1995) Life on the Screen, Simon and Shuster.

Velez E. (2014) 'Intimate Publics and Ephemerality. Snapchat: A Case Study', The Second Shift, URL (accessed January 2015) http://www.secondshiftblog.com/2014/09/intimatepublics-and-ephemerality-snapchat-a-case-study/

Walker K (2000) "'It's Difficult to Hide It": The Presentation of Self on Internet Home Pages', Qualitative Sociology, 23 (1): pp. 99-120.

Williams P. (2006) 'Authentic Identities: Straightedge Subculture, Music and the Internet', Journal of Contemporary Ethnography, 35 (2): 173-200.

\section{Notes}

\footnotetext{
' Rayes-Goldie (2010) distinguishes between 'social privacy' and 'institutional privacy'. The latter refers to questions about access to communications by distant corporations and governments while the former concerns privacy relative to immediate and more distant social networks of internet users. Notwithstanding the importance of institutional privacy, the concern here is with more immediate, everyday experiences related to social privacy.
} 